ANNALES

POLONICI MATHEMATICI

$80(2003)$

\title{
An extension theorem for separately holomorphic functions with analytic singularities
}

by MAREK Jarnicki (Kraków) and Peter Pflug (Oldenburg)

Dedicated to Professor Józef Siciak in honour of his 70th birthday

Abstract. Let $D_{j} \subset \mathbb{C}^{k_{j}}$ be a pseudoconvex domain and let $A_{j} \subset D_{j}$ be a locally pluriregular set, $j=1, \ldots, N$. Put

$$
X:=\bigcup_{j=1}^{N} A_{1} \times \ldots \times A_{j-1} \times D_{j} \times A_{j+1} \times \ldots \times A_{N} \subset \mathbb{C}^{k_{1}+\ldots+k_{N}} .
$$

Let $U$ be an open connected neighborhood of $X$ and let $M \varsubsetneqq U$ be an analytic subset. Then there exists an analytic subset $\widehat{M}$ of the "envelope of holomorphy" $\widehat{X}$ of $X$ with $\widehat{M} \cap X \subset M$ such that for every function $f$ separately holomorphic on $X \backslash M$ there exists an $\widehat{f}$ holomorphic on $\widehat{X} \backslash \widehat{M}$ with $\left.\widehat{f}\right|_{X \backslash M}=f$. The result generalizes special cases which were studied in [Ökt 1998], [Ökt 1999], [Sic 2001], and [Jar-Pfl 2001].

1. Introduction. Main theorem. Let $N \in \mathbb{N}, N \geq 2$, and let

$$
\emptyset \neq A_{j} \subset D_{j} \subset \mathbb{C}^{k_{j}},
$$

where $D_{j}$ is a domain, $j=1, \ldots, N$. We define an $N$-fold cross

$$
\begin{aligned}
X & :=\mathbb{X}\left(A_{1}, \ldots, A_{N} ; D_{1}, \ldots, D_{N}\right) \\
& :=\bigcup_{j=1}^{N} A_{1} \times \ldots \times A_{j-1} \times D_{j} \times A_{j+1} \times \ldots \times A_{N} \subset \mathbb{C}^{k_{1}+\ldots+k_{N}} .
\end{aligned}
$$

Observe that $X$ is connected.

Let $\Omega \subset \mathbb{C}^{n}$ be an open set and let $A \subset \Omega$. Put

$$
h_{A, \Omega}:=\sup \{u: u \in \mathcal{P} \mathcal{S H}(\Omega), u \leq 1 \text { on } \Omega, u \leq 0 \text { on } A\} \text {, }
$$

2000 Mathematics Subject Classification: 32D15, 32D10.

Key words and phrases: separately holomorphic, pluriregular, holomorphic extension. Research of M. Jarnicki partially supported by the KBN grant No. 5 P03A 03321.

Research of P. Pflug partially supported by the Niedersächsisches Ministerium für Wissenschaft und Kultur, Az. 15.3 - 50 113(55) PL. 
where $\mathcal{P S H}(\Omega)$ denotes the set of all functions plurisubharmonic on $\Omega$. Define

$$
\omega_{A, \Omega}:=\lim _{k \rightarrow \infty} h_{A \cap \Omega_{k}, \Omega_{k}}^{*},
$$

where $\left(\Omega_{k}\right)_{k=1}^{\infty}$ is a sequence of relatively compact open sets $\Omega_{k} \subset \Omega_{k+1} \subset \subset \Omega$ with $\bigcup_{k=1}^{\infty} \Omega_{k}=\Omega$, and $h^{*}$ denotes the upper semicontinuous regularization of $h$. Observe that the definition is independent of the exhausting sequence $\left(\Omega_{k}\right)_{k=1}^{\infty}$ chosen. Moreover, $\omega_{A, \Omega} \in \mathcal{P S H}(\Omega)$.

For an $N$-fold cross $X=\mathbb{X}\left(A_{1}, \ldots, A_{N} ; D_{1}, \ldots, D_{N}\right)$ put

$$
\widehat{X}:=\left\{\left(z_{1}, \ldots, z_{N}\right) \in D_{1} \times \ldots \times D_{N}: \sum_{j=1}^{N} \omega_{A_{j}, D_{j}}\left(z_{j}\right)<1\right\} ;
$$

notice that $\widehat{X}$ may be empty. Observe that $\widehat{X}$ is pseudoconvex if $D_{1}, \ldots, D_{N}$ are pseudoconvex domains.

We say that a subset $\emptyset \neq A \subset \mathbb{C}^{n}$ is locally pluriregular if $h_{A \cap \Omega, \Omega}^{*}(a)=0$ for any $a \in A$ and for any open neighborhood $\Omega$ of $a$ (in particular, $A \cap \Omega$ is non-pluripolar).

Note that if $A_{1}, \ldots, A_{N}$ are locally pluriregular, then $X \subset \widehat{X}$ and, moreover, $\widehat{X}$ is connected (Lemma 4 ).

Let $U$ be a connected neighborhood of $X$ and let $M \varsubsetneqq U$ be an analytic subset ( $M$ may be empty). We say that a function $f: X \backslash M \rightarrow \mathbb{C}$ is separately holomorphic $\left(f \in \mathcal{O}_{\mathrm{s}}(X \backslash M)\right)$ if for any $\left(a_{1}, \ldots, a_{N}\right) \in A_{1} \times \ldots \times A_{N}$ and $j \in\{1, \ldots, N\}$ the function $f\left(a_{1}, \ldots, a_{j-1}, \cdot, a_{j+1}, \ldots, a_{N}\right)$ is holomorphic in the domain $\left\{z_{j} \in D_{j}:\left(a_{1}, \ldots, a_{j-1}, z_{j}, a_{j+1}, \ldots, a_{N}\right) \notin M\right\}$.

The main result of our paper is the following extension theorem for separately holomorphic functions $\left({ }^{1}\right)$.

Main Theorem. Let $D_{j} \subset \mathbb{C}^{k_{j}}$ be a pseudoconvex domain and let $A_{j} \subset D_{j}$ be a locally pluriregular set, $j=1, \ldots, N$. Let $M \varsubsetneqq U$ be an analytic subset of an open connected neighborhood $U$ of $X=\mathbb{X}\left(A_{1}, \ldots, A_{N}\right.$; $\left.D_{1}, \ldots, D_{N}\right)(M$ may be empty). Then there exists a pure one-codimensional analytic subset $\widehat{M} \subset \widehat{X}$ such that:

- $\widehat{M} \cap U_{0} \subset M$ for an open neighborhood $U_{0}$ of $X, U_{0} \subset U$,

- for every $f \in \mathcal{O}_{\mathrm{s}}(X \backslash M)$ there exists exactly one $\widehat{f} \in \mathcal{O}(\widehat{X} \backslash \widehat{M})$ with $\left.\widehat{f}\right|_{X \backslash M}=f$.

Moreover, if $U=\widehat{X}$, then we can take $\widehat{M}:=$ the union of all onecodimensional irreducible components of $M$.

The proof will be given in Sections 3 (the case $U=\widehat{X}$ ) and 4 (the general case).

$\left({ }^{1}\right)$ We thank Professor Józef Siciak for turning our attention to this problem. 
Remark. Notice that the Main Theorem may be generalized to the case where $D_{j}$ is a Riemann-Stein domain over $\mathbb{C}^{k_{j}}, j=1, \ldots, N$.

Observe that in the case $M=\emptyset, N=2$, the Main Theorem is nothing else than the following cross theorem.

Theorem 1 (cf. [Ale-Zer 2001]). Let $D \subset \mathbb{C}^{p}, G \subset \mathbb{C}^{q}$ be pseudoconvex domains and let $A \subset D, B \subset G$ be locally pluriregular. Put $X:=$ $\mathbb{X}(A, B ; D, G)$. Then for any $f \in \mathcal{O}_{\mathrm{s}}(X)$ there exists exactly one $\widehat{f} \in \mathcal{O}(\widehat{X})$ with $\widehat{f}=f$ on $X$.

REmark. (a) Let $M=\emptyset$. There is a long list of papers discussing the case $N=2$ (under various assumptions): [Sic 1969], [Zah 1976], [Sic 1981], [Shi 1989], [Ngu-Zer 1991], [Ngu 1997], [Ale-Zer 2001]. The case $N \geq 2$, $k_{1}=\ldots=k_{N}=1$ can be found in [Sic 1981]. The general case $N \geq 2$, $k_{1}, \ldots, k_{N} \geq 1$ was solved in [Ngu-Zer 1995] $\left({ }^{2}\right)$.

(b) Let $M \neq \emptyset$. J. Siciak [Sic 2001] solved the case: $N \geq 2, k_{1}=\ldots=$ $k_{N}=1, D_{1}=\ldots=D_{N}=\mathbb{C}, M=P^{-1}(0)$, where $P$ is a non-zero polynomial of $N$ complex variables. The special subcase $N=2, P(z, w):=z-w$ had been studied in [Ökt 1998]. The general case for $N=2, k_{1}=k_{2}=1$ was solved in [Jar-Pfl 2001]; see also [Ökt 1999] for a partial discussion of the case $N=2, k_{1}, k_{2} \geq 1$.

The case where the singular set $M$ is a pluripolar relatively closed subset of $U$ is studied in [Jar-Pfl 2003].

2. Auxiliary results. The following lemma gathers a few standard results, which will be frequently used in what follows.

Lemma 2 (cf. [Kli 1991], [Jar-Pfl 2000, §3.5]). (a) Let $\Omega \subset \mathbb{C}^{n}$ be a bounded open set and let $A \subset \Omega$. Then:

- If $P \subset \mathbb{C}^{n}$ is pluripolar, then $h_{A \backslash P, \Omega}^{*}=h_{A, \Omega}^{*}$.

- $h_{A_{k} \cap \Omega_{k}, \Omega_{k}} \searrow h_{A, \Omega}^{*}$ (pointwise on $\Omega$ ) for any sequence of open sets $\Omega_{k} \nearrow \Omega$ and any sequence $A_{k} \nearrow A$.

- $\omega_{A, \Omega}=h_{A, \Omega}^{*}$.

- The following conditions are equivalent: for any connected component $S$ of $\Omega$ the set $A \cap S$ is non-pluripolar, and $h_{A, \Omega}^{*}(z)<1$ for any $z \in \Omega$.

- If $A$ is non-pluripolar, $0<\alpha<1$, and $\Omega_{\alpha}:=\left\{z \in \Omega: h_{A, \Omega}^{*}(z)<\alpha\right\}$, then for any connected component $S$ of $\Omega_{\alpha}$ the set $A \cap S$ is non-pluripolar (in particular, $A \cap S \neq \emptyset$ ).

- If $A$ is locally pluriregular, $0<\alpha \leq 1$, and $\Omega_{\alpha}$ is as above, then $h_{A, \Omega}^{*}=\alpha h_{A, \Omega_{\alpha}}^{*}$ on $\Omega_{\alpha}$.

$\left(^{2}\right)$ We thank Professor Nguyen Thanh Van for calling our attention to that paper. 
(b) Let $\Omega \subset \mathbb{C}^{n}$ be an open set and let $A \subset \Omega$. Then:

- $\omega_{A, \Omega} \in \mathcal{P S H}(\Omega)$.

- If $A$ is locally pluriregular, then $\omega_{A, \Omega}(a)=0$ for any $a \in A$.

- If $P \subset \mathbb{C}^{n}$ is pluripolar, then $\omega_{A \backslash P, \Omega}=\omega_{A, \Omega}$.

- If $A$ is locally pluriregular and $P \subset \mathbb{C}^{n}$ is pluripolar, then $A \backslash P$ is locally pluriregular.

Moreover, we get:

Lemma 3. (a) Let $A_{j} \subset \mathbb{C}^{k_{j}}$ be locally pluriregular, $j=1, \ldots, N$. Then $A_{1} \times \ldots \times A_{N}$ is locally pluriregular.

(b) Let $A_{j} \subset \Omega_{j} \Subset \mathbb{C}^{k_{j}}, \Omega_{j}$ a domain, $A_{j}$ locally pluriregular, $j=$ $1, \ldots, N, N \geq 2$. Put

$$
\Omega:=\left\{\left(z_{1}, \ldots, z_{N}\right) \in \Omega_{1} \times \ldots \times \Omega_{N}: \sum_{j=1}^{N} h_{A_{j}, \Omega_{j}}^{*}\left(z_{j}\right)<1\right\}
$$

(observe that $A_{1} \times \ldots \times A_{N} \subset \Omega$ ). Then

$$
h_{A_{1} \times \ldots \times A_{N}, \Omega}^{*}=\sum_{j=1}^{N} h_{A_{j}, \Omega_{j}}^{*} \quad \text { on } \Omega .
$$

Proof. (a) is an immediate consequence of the product property for the relatively extremal function

$$
h_{B_{1} \times \ldots \times B_{N}, \Omega_{1} \times \ldots \times \Omega_{N}}^{*}=\max \left\{h_{B_{j}, \Omega_{j}}^{*}: j=1, \ldots, N\right\} ;
$$

cf. [Ngu-Sic 1991].

(b) First observe that

$$
\sum_{j=1}^{N} h_{A_{j}, \Omega_{j}}^{*} \leq h_{A_{1} \times \ldots \times A_{N}, \Omega}^{*} \quad \text { on } \Omega .
$$

To get the opposite inequality we proceed by induction on $N \geq 2$.

Let $N=2$. The proof of this step is taken from [Sic 1981]. For the reader's convenience we repeat the details.

Put $u:=h_{A_{1} \times A_{2}, \Omega}^{*} \in \mathcal{P} \mathcal{S H}(\Omega)$ and fix a point $\left(a_{1}, a_{2}\right) \in \Omega$. If $a_{1} \in A_{1}$ (thus $h_{A_{1}, \Omega_{1}}^{*}\left(a_{1}\right)=0$ ), then $u\left(a_{1}, \cdot\right) \in \mathcal{P} \mathcal{S H}\left(\Omega_{2}\right)$ with $u\left(a_{1}, \cdot\right) \leq 1$ and $u\left(a_{1}, \cdot\right) \leq 0$ on $A_{2}$. Therefore,

$$
u\left(a_{1}, \cdot\right) \leq h_{A_{2}, \Omega_{2}}^{*}=h_{A_{1}, \Omega_{1}}^{*}\left(a_{1}\right)+h_{A_{2}, \Omega_{2}}^{*} \quad \text { on } \Omega_{2} .
$$

In particular, $u\left(a_{1}, a_{2}\right) \leq h_{A_{1}, \Omega_{1}}^{*}\left(a_{1}\right)+h_{A_{2}, \Omega_{2}}^{*}\left(a_{2}\right)$.

Observe that the same argument shows that if $a_{2} \in A_{2}$, then $u\left(\cdot, a_{2}\right) \leq$ $h_{A_{1}, \Omega_{1}}^{*}$ on $\Omega_{1}$. 
If $a_{1} \notin A_{1}$, then $h_{A_{1}, \Omega_{1}}^{*}\left(a_{1}\right)+h_{A_{2}, \Omega_{2}}^{*}\left(a_{2}\right)<1$ and therefore $\alpha:=1-$ $h_{A_{1}, \Omega_{1}}^{*}\left(a_{1}\right) \in(0,1]$. Put

$$
\left(\Omega_{2}\right)_{\alpha}:=\left\{z_{2} \in \Omega_{2}: h_{A_{2}, \Omega_{2}}^{*}\left(z_{2}\right)<\alpha\right\} .
$$

It is clear that $A_{2} \subset\left(\Omega_{2}\right)_{\alpha} \ni a_{2}$. Put

$$
v:=\frac{1}{\alpha}\left(u\left(a_{1}, \cdot\right)-h_{A_{1}, \Omega_{1}}^{*}\left(a_{1}\right)\right) \in \mathcal{P} \mathcal{S H}\left(\left(\Omega_{2}\right)_{\alpha}\right) .
$$

Then $v \leq 1$ and $v \leq 0$ on $A_{2}$. Therefore, by Lemma 2(a),

$$
v \leq h_{A_{2},\left(\Omega_{2}\right)_{\alpha}}^{*}\left(a_{2}\right)=\frac{1}{\alpha} h_{A_{2}, \Omega_{2}}^{*}\left(a_{2}\right) \quad \text { on }\left(\Omega_{2}\right)_{\alpha} .
$$

Consequently, $u\left(a_{1}, a_{2}\right) \leq h_{A_{1}, \Omega_{1}}^{*}\left(a_{1}\right)+h_{A_{2}, \Omega_{2}}^{*}\left(a_{2}\right)$, which finishes the proof for $N=2$.

Now, assume that the formula is true for $N-1 \geq 2$. Put

$$
\widetilde{\Omega}:=\left\{\left(z_{1}, \ldots, z_{N-1}\right) \in \Omega_{1} \times \ldots \times \Omega_{N-1}: \sum_{j=1}^{N-1} h_{A_{j}, \Omega_{j}}^{*}\left(z_{j}\right)<1\right\}
$$

and fix an arbitrary $z=\left(\widetilde{z}, z_{N}\right) \in \Omega$. Obviously, $\widetilde{z} \in \widetilde{\Omega}$. By the inductive hypothesis, we conclude that

$$
h_{A_{1} \times \ldots \times A_{N-1}, \widetilde{\Omega}}^{*}(\widetilde{z})=\sum_{j=1}^{N-1} h_{A_{j}, \Omega_{j}}^{*}\left(z_{j}\right) .
$$

Now we apply the case $N=2$ to the following situation:

$$
\Omega^{\prime}:=\left\{\left(\widetilde{w}, w_{N}\right) \in \widetilde{\Omega} \times \Omega_{N}: h_{A_{1} \times \ldots \times A_{N-1}, \widetilde{\Omega}}^{*}(\widetilde{w})+h_{A_{N}, \Omega_{N}}^{*}\left(w_{N}\right)<1\right\} .
$$

So

$$
\begin{aligned}
h_{A_{1} \times \ldots \times A_{N-1}, \widetilde{\Omega}}^{*}(\widetilde{w})+h_{A_{N}, \Omega_{N}}^{*}\left(w_{N}\right) & \\
& =h_{A_{1} \times \ldots \times A_{N}, \Omega^{\prime}}^{*}\left(\widetilde{w}, w_{N}\right), \quad\left(\widetilde{w}, w_{N}\right) \in \Omega^{\prime} .
\end{aligned}
$$

Note that $\Omega^{\prime}=\Omega$. Hence

$$
h_{A_{1} \times \ldots \times A_{N}, \Omega}^{*}\left(\widetilde{z}, z_{N}\right)=h_{A_{1} \times \ldots \times A_{N-1}, \widetilde{\Omega}}^{*}(\widetilde{z})+h_{A_{N}, \Omega_{N}}\left(z_{N}\right) \stackrel{(2)}{=} \sum_{j=1}^{N} h_{A_{j}, \Omega_{j}}^{*}\left(z_{j}\right) \text {. }
$$

Lemma 4. Let $X=\mathbb{X}\left(A_{1}, \ldots, A_{N} ; D_{1}, \ldots, D_{N}\right)$ be an $N$-fold cross as in (1). If $A_{1}, \ldots, A_{N}$ are locally pluriregular, then $\widehat{X}$ is a domain.

Proof. Using exhaustion by bounded domains we may assume that the $D_{j}$ 's are bounded.

We know that $X \subset \widehat{X}$. Let $z^{0}=\left(z_{1}^{0}, \ldots, z_{N}^{0}\right) \in \widehat{X}$ be an arbitrary point. If $\sum_{j=2}^{N} h_{A_{j}, D_{j}}^{*}\left(z_{j}^{0}\right)=0$, then $D_{1} \times\left\{\left(z_{2}^{0}, \ldots, z_{N}^{0}\right)\right\} \subset \widehat{X}$. Therefore, $z^{0}$ can be joined inside $D_{1} \times\left\{\left(z_{2}^{0}, \ldots, z_{N}^{0}\right)\right\}$ to $\left(a_{1}, z_{2}^{0}, \ldots, z_{N}^{0}\right)$ for some $a_{1} \in A_{1}$. 


$$
\text { If } \begin{aligned}
\sum_{j=2}^{N} h_{A_{j}, D_{j}}^{*}\left(z_{j}^{0}\right)=: & \varepsilon>0, \text { put } \\
\left(D_{1}\right)_{1-\varepsilon} & :=\left\{z_{1} \in D_{1}: h_{A_{1}, D_{1}}^{*}\left(z_{1}\right)<1-\varepsilon\right\} .
\end{aligned}
$$

Then, by Lemma $2(\mathrm{a})$, the connected component $S$ of $\left(D_{1}\right)_{1-\varepsilon}$ that contains $z_{1}^{0}$ intersects $A_{1}$. Therefore, $z^{0}$ can be joined inside $S \times\left\{\left(z_{2}^{0}, \ldots, z_{N}^{0}\right)\right\} \subset \widehat{X}$ to $\left(a_{1}, z_{2}^{0}, \ldots, z_{N}^{0}\right)$ for some $a_{1} \in A_{1}$.

Now we repeat the above argument for the second component of the point $\left(a_{1}, z_{2}^{0}, \ldots, z_{N}^{0}\right)$. Finally, the point $z^{0}$ can be joined inside $\widehat{X}$ to $\left(a_{1}, \ldots, a_{N}\right)$ $\in A_{1} \times \ldots \times A_{N} \subset X$. Since $X$ is connected, the proof is complete.

Lemma 5 (Identity theorems). (a) Let $\Omega \subset \mathbb{C}^{n}$ be a domain and let $A \subset \Omega$ be non-pluripolar. Then any $f \in \mathcal{O}(\Omega)$ with $\left.f\right|_{A}=0$ vanishes identically on $\Omega$.

(b) Let $D \subset \mathbb{C}^{p}, G \subset \mathbb{C}^{q}$ be domains, let $A \subset D, B \subset G$ be locally pluriregular sets, and let $X:=\mathbb{X}(A, B ; D, G)$. Let $M \varsubsetneqq U$ be an analytic subset of an open connected neighborhood $U$ of $X$. Assume that $A^{\prime} \subset A$, $B^{\prime} \subset B$ are such that:

- $A \backslash A^{\prime}$ and $B \backslash B^{\prime}$ are pluripolar (in particular, $A^{\prime}, B^{\prime}$ are also locally pluriregular $)$,

- $M_{z}:=\{w \in G:(z, w) \in M\} \neq G$ for any $z \in A^{\prime}$,

- $M^{w}:=\{z \in D:(z, w) \in M\} \neq D$ for any $w \in B^{\prime}$.

Then:

$\left(\mathrm{b}_{1}\right)$ If $f \in \mathcal{O}_{\mathrm{s}}(X \backslash M)$ and $f=0$ on $A^{\prime} \times B^{\prime} \backslash M\left({ }^{3}\right)$, then $f=0$ on $X \backslash M$.

$\left(\mathrm{b}_{2}\right)$ If $g \in \mathcal{O}(U \backslash M)$ and $g=0$ on $A^{\prime} \times B^{\prime} \backslash M$, then $g=0$ on $U \backslash M$.

Proof. (a) is obvious.

$\left(\mathrm{b}_{1}\right)$ Take a point $\left(a_{0}, b_{0}\right) \in X \backslash M$. We may assume that $a_{0} \in A$. Since $A \backslash A^{\prime}$ is pluripolar, there exists a sequence $\left(a_{k}\right)_{k=1}^{\infty} \subset A^{\prime}$ such that $a_{k} \rightarrow a_{0}$. The set $Q:=\bigcup_{k=0}^{\infty} M_{a_{k}}$ is pluripolar. Consequently, the set $B^{\prime \prime}:=$ $B^{\prime} \backslash Q$ is non-pluripolar. We have $f\left(a_{k}, w\right)=0, w \in B^{\prime \prime}, k=1,2, \ldots$ Hence $f\left(a_{0}, w\right)=0$ for any $w \in B^{\prime \prime}$. Finally, $f\left(a_{0}, w\right)=0$ on $G \backslash M_{a_{0}} \ni b_{0}$.

$\left(\mathrm{b}_{2}\right)$ Take an $a_{0} \in A^{\prime}$. Since $M_{a_{0}} \neq G$, there exists a $b_{0} \in B^{\prime} \backslash M_{a_{0}}$. Let $P=\Delta_{a_{0}}(r) \times \Delta_{b_{0}}(r) \subset U \backslash M\left(\Delta_{z_{0}}(r) \subset \mathbb{C}^{p}\right.$ denotes the polydisc with center $z_{0} \in \mathbb{C}^{p}$ and radius $\left.r>0\right)$. Then $g(\cdot, w)=0$ on $A^{\prime} \cap \Delta_{a_{0}}(r)$ for any $w \in B^{\prime} \cap \Delta_{b_{0}}(r)$. The set $A^{\prime} \cap \Delta_{a_{0}}(r)$ is non-pluripolar. Hence $g(\cdot, w)=0$ on $\Delta_{a_{0}}(r)$ for any $w \in B^{\prime} \cap \Delta_{b_{0}}(r)$. By the same argument for the second variable we get $g=0$ on $P$ and, consequently, on $U \backslash M$.

$\left({ }^{3}\right)$ Here and below, to simplify notation we write $P \times Q \backslash M$ instead of $(P \times Q) \backslash M$. 
3. Proof of the Main Theorem in the case where $U=\widehat{X}$. We proceed by several reduction steps. First observe that, by Lemma $5(\mathrm{a})$, the function $\widehat{f}$ is uniquely determined (if it exists).

SteP 1. To prove the Main Theorem for $M \neq \emptyset$ it suffices to consider the case where $M$ is pure one-codimensional.

Proof. Since $\widehat{X}$ is pseudoconvex, the arbitrary analytic set $M \subset \widehat{X}$ can be written as

$$
M=\left\{z \in \widehat{X}: g_{1}(z)=\ldots=g_{k}(z)=0\right\},
$$

where $g_{j} \in \mathcal{O}(\widehat{X}), g_{j} \not \equiv 0, j=1, \ldots, k$. Then $M_{j}:=g_{j}^{-1}(0)$ is pure onecodimensional.

Take an $f \in \mathcal{O}_{\mathrm{s}}(X \backslash M)$. Observe that $f_{j}:=\left.f\right|_{X \backslash M_{j}} \in \mathcal{O}_{\mathrm{s}}\left(X \backslash M_{j}\right)$. By the reduction assumption there exists an $\widehat{f}_{j} \in \mathcal{O}\left(\widehat{X} \backslash M_{j}\right)$ such that $\widehat{f}_{j}=f$ on $X \backslash M_{j}$. In view of Lemma $5(\mathrm{a})$, gluing the functions $\left(\widehat{f}_{j}\right)_{j=1}^{k}$ leads to an $\widehat{f} \in \mathcal{O}(\widehat{X} \backslash M)$ with $\widehat{f}=\widehat{f}_{j}$ on $\widehat{X} \backslash M_{j}, j=1, \ldots, k$. Therefore, $\widehat{f}=f$ on $X \backslash M$.

Finally, since $\operatorname{codim}(M \backslash \widehat{M}) \geq 2$, the function $\widehat{f}$ extends holomorphically across $M \backslash \widehat{M}$.

From now on we assume that $M$ is empty or pure one-codimensional.

SteP 2. To prove the Main Theorem it suffices to consider the case where $M$ is empty or pure one-codimensional and $D_{1}, \ldots, D_{N}$ are bounded pseudoconvex.

Proof. Let $D_{1}, \ldots, D_{N}$ be arbitrary pseudoconvex domains, and let $D_{j, k} \nearrow D_{j}, D_{j, k} \Subset D_{j}$, where $D_{j, k}$ are pseudoconvex domains with $A_{j, k}:=$ $A_{j} \cap D_{j, k} \neq \emptyset$. Observe that all the $A_{j, k}$ 's are locally pluriregular. Put

$$
X_{k}:=\mathbb{X}\left(A_{1, k}, \ldots, A_{N, k} ; D_{1, k}, \ldots, D_{N, k}\right) \subset X ;
$$

note that $\widehat{X}_{k} \nearrow \widehat{X}$.

Let $f \in \mathcal{O}_{\mathrm{s}}(X \backslash M)$ be given. By the reduction assumption, for each $k$ there exists an $\widehat{f}_{k} \in \mathcal{O}\left(\widehat{X}_{k} \backslash M\right)$ with $\widehat{f}_{k}=f$ on $X_{k} \backslash M$. By Lemma $5(\mathrm{a})$, $\widehat{f}_{k+1}=\widehat{f}_{k}$ on $\widehat{X}_{k} \backslash M$. Therefore, gluing the $\widehat{f}_{k}$ 's, we obtain an $\widehat{f} \in \mathcal{O}(\widehat{X} \backslash M)$ with $\widehat{f}=f$ on $X \backslash M$.

From now on we assume that $M$ is empty or pure one-codimensional and $D_{1}, \ldots, D_{N}$ are bounded pseudoconvex.

STEP 3. To prove the Main Theorem it suffices to consider the case $N=2$.

Remark. By Theorem 1, Step 3 finishes the proof of the Main Theorem for $M=\emptyset$. 
Proof of Step 3. We proceed by induction on $N \geq 2$. Suppose that the Main Theorem is true for $N-1 \geq 2$. We have to discuss the case of an $N$ fold cross $X=\mathbb{X}\left(A_{1}, \ldots, A_{N} ; D_{1}, \ldots, D_{N}\right)$, where $D_{1}, \ldots, D_{N}$ are bounded pseudoconvex. Let $M \subset \widehat{X}$ be empty or pure one-codimensional.

Let $f \in \mathcal{O}_{\mathrm{s}}(X \backslash M)$. Observe that

$$
X=\left(Y \times A_{N}\right) \cup\left(\widehat{A} \times D_{N}\right),
$$

where

$$
Y:=\mathbb{X}\left(A_{1}, \ldots, A_{N-1} ; D_{1}, \ldots, D_{N-1}\right), \quad \widehat{A}:=A_{1} \times \ldots \times A_{N-1} .
$$

We also mention that for any $a_{N} \in A_{N}$ we have

$$
\left\{\left(z_{1}, \ldots, z_{N-1}\right) \in \mathbb{C}^{k_{1}} \times \ldots \times \mathbb{C}^{k_{N-1}}:\left(z_{1}, \ldots, z_{N-1}, a_{N}\right) \in \widehat{X}\right\}=\widehat{Y} .
$$

Now fix an $a_{N} \in A_{N}$ such that

$$
M_{a_{N}}:=\left\{\left(z_{1}, \ldots, z_{N-1}\right) \in \widehat{Y}:\left(z_{1}, \ldots, z_{N-1}, a_{N}\right) \in M\right\} \varsubsetneqq \widehat{Y} ;
$$

in particular, $M_{a_{N}}$ is empty or one-codimensional (in $\widehat{Y}$ ). Recall that $A_{1}$, $\ldots, A_{N-1}$ are locally pluriregular. By the inductive assumption there exists an $\widehat{f}_{a_{N}} \in \mathcal{O}\left(\widehat{Y} \backslash M_{a_{N}}\right)$ with $\widehat{f}_{a_{N}}=f\left(\cdot, a_{N}\right)$ on $Y \backslash M_{a_{N}}$.

To continue define the following 2-fold cross:

$$
Z:=\mathbb{X}\left(\widehat{A}, A_{N} ; \widehat{Y}, D_{N}\right) .
$$

Notice that $Z$ satisfies all the properties for the case $N=2: \widehat{Y}, D_{N}$ are bounded pseudoconvex domains, $\widehat{A} \subset \widehat{Y}, A_{N} \subset D_{N}$ are locally pluriregular.

By Lemma 3, we have

$$
\widehat{Z}=\left\{\left(\widehat{z}, z_{N}\right) \in \widehat{Y} \times D_{N}: h_{\widehat{A}, \widehat{Y}}^{*}(\widehat{z})+h_{A_{N}, D_{N}}^{*}\left(z_{N}\right)<1\right\}=\widehat{X} .
$$

Define $\tilde{f}: Z \backslash M \rightarrow \mathbb{C}$ by

$$
\widetilde{f}(z)=\widetilde{f}\left(\widehat{z}, z_{N}\right):= \begin{cases}\widehat{f}_{z_{N}}(\widehat{z}) & \text { if } z \in \widehat{Y} \times A_{N}, \\ f(z) & \text { if } z \in \widehat{A} \times D_{N} .\end{cases}
$$

Obviously, $\tilde{f}$ is well defined and therefore $\tilde{f} \in \mathcal{O}_{\mathrm{s}}(Z \backslash M)$.

Using the case $N=2$, we find another function $\widehat{f} \in \mathcal{O}(\widehat{Z} \backslash M)$ with $\widehat{f}=\widetilde{f}$ on $Z \backslash M$. Recall that $\widehat{Z}=\widehat{X}$. Hence $\widehat{f}=f$ on $X \backslash M$.

What remains is to prove the case $N=2$ and $M \neq \emptyset$. From now on we simplify our notation and consider the following configuration:

Let $A \subset D \Subset \mathbb{C}^{p}, B \subset G \Subset \mathbb{C}^{q}$, where $D, G$ are bounded pseudoconvex domains, $A, B$ are locally pluriregular. Put, as always,

$$
X:=\mathbb{X}(A, B ; D, G), \quad \widehat{X}:=\left\{(z, w) \in D \times G: h_{A, D}^{*}(z)+h_{B, G}^{*}(w)<1\right\} .
$$

Moreover, let $M$ be a pure one-codimensional analytic subset of $\widehat{X}$. 
We want to show that any $f \in \mathcal{O}_{\mathrm{s}}(X \backslash M)$ extends holomorphically to $\widehat{X} \backslash M$.

SteP 4. Let $X, M$, and $f$ be as above. Let $\left(D_{j}\right)_{j=1}^{\infty},\left(G_{j}\right)_{j=1}^{\infty}$ be sequences of pseudoconvex domains, $D_{j} \Subset D, G_{j} \Subset G$, with $D_{j} \nearrow D, G_{j} \nearrow G$. Moreover, let $A^{\prime} \subset A, B^{\prime} \subset B$ be such that $A \backslash A^{\prime}, B \backslash B^{\prime}$ are pluripolar, and $A^{\prime} \cap D_{j} \neq \emptyset, B^{\prime} \cap G_{j} \neq \emptyset, j \in \mathbb{N}$. For each $j \in \mathbb{N}$ assume that for any $(a, b) \in\left(A^{\prime} \cap D_{j}\right) \times\left(B^{\prime} \cap G_{j}\right)$ there exist polydiscs $\Delta_{a}\left(r_{a, j}\right) \subset D_{j}$, $\Delta_{b}\left(s_{b, j}\right) \subset G_{j}$ with $\left(\Delta_{a}\left(r_{a, j}\right) \times G_{j}\right) \cup\left(D_{j} \times \Delta_{b}\left(s_{b, j}\right)\right) \subset \widehat{X}$, and functions $f_{a, j} \in \mathcal{O}\left(\Delta_{a}\left(r_{a, j}\right) \times G_{j} \backslash M\right), f^{b, j} \in \mathcal{O}\left(D_{j} \times \Delta_{b}\left(s_{b, j}\right) \backslash M\right)$ such that

- $f_{a, j}=f$ on $\left(A^{\prime} \cap \Delta_{a}\left(r_{a, j}\right)\right) \times G_{j} \backslash M$,

- $f^{b, j}=f$ on $D_{j} \times\left(B^{\prime} \cap \Delta_{b}\left(s_{b, j}\right)\right) \backslash M$.

Then there exists an $\widehat{f} \in \mathcal{O}(\widehat{X} \backslash M)$ with $\widehat{f}=f$ on $X \backslash M$.

Proof. Fix a $j \in \mathbb{N}$. Put

$$
\begin{aligned}
\widetilde{U}_{j} & :=\bigcup_{\substack{a \in A^{\prime} \cap D_{j} \\
b \in B^{\prime} \cap G_{j}}}\left(\Delta_{a}\left(r_{a, j}\right) \times G_{j}\right) \cup\left(D_{j} \times \Delta_{b}\left(s_{b, j}\right)\right), \\
X_{j} & :=\left(\left(A \cap D_{j}\right) \times G_{j}\right) \cup\left(D_{j} \times\left(B \cap G_{j}\right)\right) .
\end{aligned}
$$

Note that

$$
X_{j}^{\prime}:=\left(\left(A^{\prime} \cap D_{j}\right) \times G_{j}\right) \cup\left(D_{j} \times\left(B^{\prime} \cap G_{j}\right)\right) \subset \widetilde{U}_{j} .
$$

We wish to glue the functions $\left(f_{a, j}\right)_{a \in A^{\prime} \cap D_{j}}$ and $\left(f^{b, j}\right)_{b \in B^{\prime} \cap G_{j}}$ to obtain a global holomorphic function $f_{j}$ on $\widetilde{U}_{j} \backslash M$. Let $a \in A^{\prime} \cap D_{j}, b \in B^{\prime} \cap G_{j}$. Observe that

$$
\begin{array}{ll}
f_{a, j}=f & \text { on }\left(A^{\prime} \cap \Delta_{a}\left(r_{a, j}\right)\right) \times G_{j} \backslash M, \\
f^{b, j}=f & \text { on } D_{j} \times\left(B^{\prime} \cap \Delta_{b}\left(s_{b, j}\right)\right) \backslash M .
\end{array}
$$

Thus $f_{a, j}=f^{b, j}$ on $\left(A^{\prime} \cap \Delta_{a}\left(r_{a, j}\right)\right) \times\left(B^{\prime} \cap \Delta_{b}\left(s_{b, j}\right)\right) \backslash M$. Applying Lemma $5(\mathrm{a})$, we conclude that

$$
f_{a, j}=f^{b, j} \quad \text { on }\left(\Delta_{a}\left(r_{a, j}\right) \times \Delta_{b}\left(s_{b, j}\right)\right) \backslash M .
$$

Now let $a^{\prime}, a^{\prime \prime} \in A^{\prime} \cap D_{j}$ be such that $\Delta_{a^{\prime}}\left(r_{a^{\prime}, j}\right) \cap \Delta_{a^{\prime \prime}}\left(r_{a^{\prime \prime}, j}\right) \neq \emptyset$. Fix a $b \in B^{\prime} \cap G_{j}$. We know that $f_{a^{\prime}, j}=f^{b, j}=f_{a^{\prime \prime}, j}$ on $\left(\Delta_{a^{\prime}}\left(r_{a^{\prime}, j}\right) \cap \Delta_{a^{\prime \prime}}\left(r_{a^{\prime \prime}, j}\right)\right) \times$ $\Delta_{b}\left(r_{b, j}\right) \backslash M$. Hence, by the identity principle, we conclude that $f_{a^{\prime}, j}=f_{a^{\prime \prime}, j}$ on $\left(\Delta_{a^{\prime}}\left(r_{a^{\prime}, j}\right) \cap \Delta_{a^{\prime \prime}}\left(r_{a^{\prime \prime}, j}\right)\right) \times G_{j} \backslash M$. The same argument works for $b^{\prime}, b^{\prime \prime} \in$ $B^{\prime} \cap G_{j}$. Consequently, we obtain a function $f_{j} \in \mathcal{O}\left(\widetilde{U}_{j} \backslash M\right)$ with $f_{j}=f$ on $X_{j}^{\prime} \backslash M$.

Let $U_{j}$ be the connected component of $\widetilde{U}_{j} \cap \widehat{X}_{j}^{\prime}$ with $X_{j}^{\prime} \subset U_{j}$. Thus we have $f_{j} \in \mathcal{O}\left(U_{j} \backslash M\right)$ with $f_{j}=f$ on $X_{j}^{\prime} \backslash M$. 
Recall that $X_{j}^{\prime} \subset U_{j} \subset \widehat{X}_{j}^{\prime}$. We claim that the envelope of holomorphy of $U_{j}$ coincides with $\widehat{X}_{j}^{\prime}$. In fact, let $h \in \mathcal{O}\left(U_{j}\right)$; then $\left.h\right|_{X_{j}^{\prime}} \in \mathcal{O}_{\mathrm{s}}\left(X_{j}^{\prime}\right)$. So, by Theorem 1 , there exists an $\widehat{h} \in \mathcal{O}\left(\widehat{X}_{j}^{\prime}\right)$ with $\widehat{h}=h$ on $X_{j}^{\prime}$. Lemma $5\left(\mathrm{~b}_{2}\right)$ implies that $\widehat{h}=h$ on $U_{j}$.

Applying the Grauert-Remmert theorem (cf. [Jar-Pfl 2000, Th. 3.4.7]), we find a function $\widehat{f}_{j} \in \mathcal{O}\left(\widehat{X}_{j}^{\prime} \backslash M\right)$ with $\widehat{f}_{j}=f_{j}$ on $U_{j} \backslash M$. In particular, $\widehat{f}_{j}=f$ on $X_{j}^{\prime} \backslash M$.

Since $A \backslash A^{\prime}, B \backslash B^{\prime}$ are pluripolar, we get

$$
\begin{aligned}
\widehat{X}_{j}^{\prime} & =\left\{(z, w) \in D_{j} \times G_{j}: h_{A^{\prime} \cap D_{j}, D_{j}}^{*}(z)+h_{B^{\prime} \cap G_{j}, G_{j}}^{*}(w)<1\right\} \\
& =\left\{(z, w) \in D_{j} \times G_{j}: h_{A \cap D_{j}, D_{j}}^{*}(z)+h_{B \cap G_{j}, G_{j}}^{*}(w)<1\right\}=\widehat{X}_{j} .
\end{aligned}
$$

So, in fact, $\widehat{f}_{j} \in \mathcal{O}\left(\widehat{X}_{j} \backslash M\right)$. Using Lemma $5\left(\mathrm{~b}_{1}\right)$, we even see that $\widehat{f}_{j}=f$ on $X_{j} \backslash M$.

Observe that $\bigcup_{j=1}^{\infty} X_{j}=X, \widehat{X}_{j} \subset \widehat{X}_{j+1}$, and $\bigcup_{j=1}^{\infty} \widehat{X}_{j}=\widehat{X}$. Using again Lemma 5 (a), by gluing the $\widehat{f}_{j}$ 's, we get a function $\widehat{f} \in \mathcal{O}(\widehat{X} \backslash M)$ with $\widehat{f}=f$ on $X \backslash M$.

To apply Step 4 we introduce the following condition $(*)$. Let $\varrho>0$, $0<r<R$. Put

$$
\Omega:=\Delta_{a_{0}}(\varrho) \times \Delta_{b_{0}}(R) \subset \mathbb{C}^{p} \times \mathbb{C}^{q}, \quad \widetilde{\Omega}:=\Delta_{a_{0}}(\varrho) \times \Delta_{b_{0}}(r) \subset \mathbb{C}^{p} \times \mathbb{C}^{q} .
$$

Let $A \subset \Delta_{a_{0}}(\varrho) \subset \mathbb{C}^{p}$ be locally pluriregular, $a_{0} \in A$, and let $M$ be a pure one-codimensional analytic subset of $\Omega$ with $M \cap \widetilde{\Omega}=\emptyset$. Put $M_{a}:=\{w \in$ $\left.\Delta_{b_{0}}(R):(a, w) \in M\right\}, a \in A$. Condition (*) reads:

(*) For any $R^{\prime} \in(r, R)$ there exists $\varrho^{\prime} \in(0, \varrho)$ such that for any function $f \in \mathcal{O}(\widetilde{\Omega})$ with $f(a, \cdot) \in \mathcal{O}\left(\Delta_{b_{0}}(R) \backslash M_{a}\right), a \in A$, there exists an extension $\widehat{f} \in \mathcal{O}\left(\Delta_{a_{0}}\left(\varrho^{\prime}\right) \times \Delta_{b_{0}}\left(R^{\prime}\right) \backslash M\right)$ with $\widehat{f}=f$ on $\Delta_{a_{0}}\left(\varrho^{\prime}\right) \times$ $\Delta_{b_{0}}(r)$.

STEP 5. If condition $(*)$ holds, then the assumptions of Step 4 are satisfied.

Proof. Take $X, M, f \in \mathcal{O}_{\mathrm{s}}(X \backslash M)$ as is in Step 4. Define

$$
A^{\prime}:=\left\{a \in A: M_{a} \neq G\right\}, \quad B^{\prime}:=\left\{a \in B: M^{b} \neq D\right\},
$$

where $M_{a}:=\{w \in G:(a, w) \in M\}, M^{b}:=\{z \in D:(z, b) \in M\}$. It is clear that $A \backslash A^{\prime}, B \backslash B^{\prime}$ are pluripolar.

Let $\left(D_{j}\right)_{j=1}^{\infty},\left(G_{j}\right)_{j=1}^{\infty}$ be approximation sequences: $D_{j} \Subset D_{j+1} \Subset D$, $G_{j} \Subset G_{j+1} \Subset G, D_{j} \nearrow D, G_{j} \nearrow G, A^{\prime} \cap D_{j} \neq \emptyset$, and $B^{\prime} \cap G_{j} \neq \emptyset, j \in \mathbb{N}$. 
Fix $j \in \mathbb{N}, a \in A^{\prime} \cap D_{j}$ and let $\Omega_{j}$ be the set of all $b \in G_{j+1}$ such that there exist a polydisc $\Delta_{(a, b)}\left(r_{b}\right) \subset D_{j} \times G_{j+1}$ and a function $\widetilde{f}_{b} \in \mathcal{O}\left(\Delta_{(a, b)}\left(r_{b}\right) \backslash M\right)$ with $\widetilde{f}_{b}=f$ on $\left(A \cap \Delta_{a}\left(r_{b}\right)\right) \times \Delta_{b}\left(r_{b}\right) \backslash M$.

It is clear that $\Omega_{j}$ is open. Observe that $\Omega_{j} \neq \emptyset$. Indeed, as $B \cap G_{j} \backslash M_{a}$ $\neq \emptyset$, we can choose a point $b \in B \cap G_{j} \backslash M_{a}$. Therefore there is a polydisc $\Delta_{(a, b)}(r) \subset D_{j} \times G_{j} \backslash M$. Put

$$
Y:=\mathbb{X}\left(A \cap \Delta_{a}(r), B \cap \Delta_{b}(r) ; \Delta_{a}(r), \Delta_{b}(r)\right) .
$$

By Theorem 1, we find $r_{b} \in(0, r)$ and $\widetilde{f}_{b} \in \mathcal{O}\left(\Delta_{(a, b)}\left(r_{b}\right)\right)$ with $\widetilde{f}_{b}=f$ on $\Delta_{(a, b)}\left(r_{b}\right) \cap Y \supset\left(A \cap \Delta_{a}\left(r_{b}\right)\right) \times \Delta_{b}\left(r_{b}\right)$. Consequently, $b \in \Omega_{j}$.

Moreover, $\Omega_{j}$ is relatively closed in $G_{j+1}$. Indeed, let $c$ be an accumulation point of $\Omega_{j}$ in $G_{j+1}$ and let $\Delta_{c}(3 R) \subset G_{j+1}$. Take a point $b \in$ $\Omega_{j} \cap \Delta_{c}(R) \backslash M_{a}$ and let $r \in\left(0, r_{b}\right], r<2 R$, be such that $\Delta_{(a, b)}(r) \cap M=\emptyset$. Observe that $\widetilde{f}_{b} \in \mathcal{O}\left(\Delta_{(a, b)}(r)\right)$ and $\widetilde{f}_{b}(z, \cdot)=f(z, \cdot) \in \mathcal{O}\left(\Delta_{b}(2 R) \backslash M_{z}\right)$ for any $z \in A \cap \Delta_{a}(r)$. Hence, by $(*)$ (with $R^{\prime}:=R$ ), there exists an extension $\widehat{\widetilde{f}}_{b} \in \mathcal{O}\left(\Delta_{a}\left(\varrho^{\prime}\right) \times \Delta_{b}(R) \backslash M\right)\left(\varrho^{\prime} \in(0, r)\right)$ such that $\widehat{\widetilde{f}}_{b}=\widetilde{f}_{b}$ on $\Delta_{(a, b)}(r)$. Take an $r_{c}>0$ so small that $\Delta_{(a, c)}\left(r_{c}\right) \subset \Delta_{a}\left(\varrho^{\prime}\right) \times \Delta_{b}(R)$ and put $\widetilde{f}_{c}:=\widehat{\widetilde{f}}_{b}$ on $\Delta_{(a, c)}\left(r_{c}\right) \backslash M$. Obviously $\widetilde{f}_{c}=\widetilde{\widetilde{f}}_{b}=f$ on $\left(A \cap \Delta_{a}\left(r_{c}\right)\right) \times \Delta_{c}\left(r_{c}\right) \backslash M$. Hence $c \in \Omega_{j}$.

Thus $\Omega_{j}=G_{j+1}$. There exists a finite set $T \subset \bar{G}_{j}$ such that

$$
\bar{G}_{j} \subset \bigcup_{b \in T} \Delta_{b}\left(r_{b}\right) .
$$

Define $r_{a, j}:=\min \left\{r_{b}: b \in T\right\}$. Take $b^{\prime}, b^{\prime \prime} \in T$ with $\Delta_{b^{\prime}}\left(r_{b^{\prime}}\right) \cap \Delta_{b^{\prime \prime}}\left(r_{b^{\prime \prime}}\right) \neq \emptyset$. Then $\widetilde{f}_{b^{\prime}}=f=\widetilde{f}_{b^{\prime \prime}}$ on $\left(A^{\prime} \cap \Delta_{a}\left(r_{a, j}\right)\right) \times\left(\Delta_{b^{\prime}}\left(r_{b^{\prime}}\right) \cap \Delta_{b^{\prime \prime}}\left(r_{b^{\prime \prime}}\right)\right) \backslash M$. Consequently, by Lemma $5(\mathrm{a}), \widetilde{f}_{b^{\prime}}=\widetilde{f}_{b^{\prime \prime}}$ on $\Delta_{a}\left(r_{a, j}\right) \times\left(\Delta_{b^{\prime}}\left(r_{b^{\prime}}\right) \cap \Delta_{b^{\prime \prime}}\left(r_{b^{\prime \prime}}\right)\right) \backslash M$. In particular, by gluing the functions $\left(\widetilde{f}_{b}\right)_{b \in T}$, we get a function $f_{a, j} \in$ $\mathcal{O}\left(\Delta_{a}\left(r_{a, j}\right) \times G_{j} \backslash M\right)$ such that $f_{a, j}=f$ on $\left(A^{\prime} \cap \Delta_{a}\left(r_{a, j}\right)\right) \times G_{j} \backslash M$.

Changing the roles of $z$ and $w$, we get $f^{b, j}, b \in B^{\prime} \cap G_{j}$.

Thus the assumptions of Step 4 are satisfied.

It remains to check $(*)$.

SteP 6. Condition (*) is always satisfied, i.e. the Main Theorem is true.

Proof. Fix a function $f \in \mathcal{O}(\widetilde{\Omega})$ such that $f(a, \cdot) \in \mathcal{O}\left(\Delta_{b_{0}}(R) \backslash M_{a}\right)$ for any $a \in A$ with $M_{a} \neq \Delta_{b_{0}}(R)$. Define

$$
\begin{aligned}
R_{0}^{*}:=\sup \left\{R^{\prime} \in[r, R): \exists_{\varrho^{\prime} \in(0, \varrho]} \exists_{\widehat{f} \in \mathcal{O}\left(\Delta_{a_{0}}\left(\varrho^{\prime}\right) \times \Delta_{b_{0}}\left(R^{\prime}\right) \backslash M\right)}:\right. \\
\left.\widehat{f}=f \text { on } \Delta_{a_{0}}\left(\varrho^{\prime}\right) \times \Delta_{b_{0}}(r)\right\} .
\end{aligned}
$$

It suffices to show that $R_{0}^{*}=R$. 
Suppose that $R_{0}^{*}<R$. Fix $R_{0}^{*}<R_{0}^{\prime}<R_{0}<R$ and choose $R^{\prime}, \varrho^{\prime}, \widehat{f}$ as in $(3)$ with $R^{\prime} \in\left[r, R_{0}^{*}\right), \sqrt[q]{R^{\prime q-1} R_{0}^{\prime}}>R_{0}^{*}$. Write $w=\left(w^{\prime}, w_{q}\right) \in \mathbb{C}^{q}=$ $\mathbb{C}^{q-1} \times \mathbb{C}$. Put $\widetilde{A}:=A \cap \Delta_{a_{0}}\left(\varrho^{\prime}\right)$.

Let $A^{\prime}$ denote the set of all $\left(a, b^{\prime}\right) \in \widetilde{A} \times \Delta_{b_{0}^{\prime}}\left(R^{\prime}\right)$ which satisfy the following condition:

$\left(\begin{array}{l}* \\ *\end{array}\right) \quad$ There exist $R^{\prime \prime} \in\left(R_{0}, R\right), \delta>0, m \in \mathbb{N}, c_{1}, \ldots, c_{m} \in \Delta_{b_{0, q}}\left(R^{\prime \prime}\right), \varepsilon>0$, and holomorphic functions $\phi_{\mu}: \Delta_{\left(a, b^{\prime}\right)}(\delta) \rightarrow \Delta_{c_{\mu}}(\varepsilon), \mu=1, \ldots, m$, such that:

- $\Delta_{\left(a, b^{\prime}\right)}(\delta) \subset \Delta_{a_{0}}\left(\varrho^{\prime}\right) \times \Delta_{b_{0}^{\prime}}\left(R^{\prime}\right)$,

- $\Delta_{c_{\mu}}(\varepsilon) \Subset \Delta_{b_{0, q}}\left(R^{\prime \prime}\right), \mu=1, \ldots, m$,

- $\bar{\Delta}_{c_{\mu}}(\varepsilon) \cap \bar{\Delta}_{c_{\nu}}(\varepsilon)=\emptyset$ for $\mu \neq \nu, \mu, \nu=1, \ldots, m$,

- $\widetilde{H}:=\Delta_{b_{0, q}}\left(R^{\prime}\right) \cap H \neq \emptyset$, where $H:=\Delta_{b_{0, q}}\left(R^{\prime \prime}\right) \backslash \bigcup_{\mu=1}^{m} \bar{\Delta}_{c_{\mu}}(\varepsilon)$,

- $\left(\Delta_{\left(a, b^{\prime}\right)}(\delta) \times \Delta_{b_{0, q}}\left(R^{\prime \prime}\right)\right) \cap M=\bigcup_{\mu=1}^{m}\left\{\left(z, w^{\prime}, \phi_{\mu}\left(z, w^{\prime}\right)\right):\left(z, w^{\prime}\right) \in\right.$ $\left.\Delta_{\left(a, b^{\prime}\right)}(\delta)\right\}$.

For any $\left(a, b^{\prime}\right) \in A^{\prime}$ define a new cross

$$
Y:=\mathbb{X}\left(\left(A \cap \Delta_{a}(\delta)\right) \times \Delta_{b^{\prime}}(\delta), \widetilde{H} ; \Delta_{\left(a, b^{\prime}\right)}(\delta), H\right) .
$$

Notice that $Y$ does not intersect $M$. In particular, $\left.\widehat{f}\right|_{Y} \in \mathcal{O}_{\mathrm{s}}(Y)$. Hence, by Theorem 1 , there exists an $\widehat{f}_{1} \in \mathcal{O}(\widehat{Y})$ with $\widehat{f}_{1}=\widehat{f}$ on $Y$. Take $R^{\prime \prime \prime} \in$ $\left(R_{0}, R^{\prime \prime}\right)$ and $\varepsilon^{\prime \prime}>\varepsilon^{\prime}>\varepsilon\left(\varepsilon^{\prime \prime} \approx \varepsilon\right)$ such that

- $\Delta_{c_{\mu}}\left(\varepsilon^{\prime \prime}\right) \Subset \Delta_{b_{0, q}}\left(R^{\prime \prime \prime}\right), \mu=1, \ldots, m$,

- $\bar{\Delta}_{c_{\mu}}\left(\varepsilon^{\prime \prime}\right) \cap \bar{\Delta}_{c_{\nu}}\left(\varepsilon^{\prime \prime}\right)=\emptyset$ for $\mu \neq \nu, \mu, \nu=1, \ldots, m$.

Then there exists $\delta^{\prime} \in(0, \delta]$ such that

- $\Delta_{\left(a, b^{\prime}\right)}\left(\delta^{\prime}\right) \times H^{\prime} \subset \widehat{Y}$, where $H^{\prime}:=\Delta_{b_{0, q}}\left(R^{\prime \prime \prime}\right) \backslash \bigcup_{\mu=1}^{m} \bar{\Delta}_{c_{\mu}}\left(\varepsilon^{\prime}\right)$.

In particular, $\widehat{f_{1}} \in \mathcal{O}\left(\Delta_{\left(a, b^{\prime}\right)}\left(\delta^{\prime}\right) \times H^{\prime}\right)$.

Fix $\mu \in\{1, \ldots, m\}$. Then $\widehat{f}_{1} \in \mathcal{O}\left(\Delta_{\left(a, b^{\prime}\right)}\left(\delta^{\prime}\right) \times\left(\Delta_{c_{\mu}}\left(\varepsilon^{\prime \prime}\right) \backslash \bar{\Delta}_{c_{\mu}}\left(\varepsilon^{\prime}\right)\right)\right)$ and $\widehat{f}_{1}\left(z, w^{\prime}, \cdot\right) \in \mathcal{O}\left(\Delta_{c_{\mu}}\left(\varepsilon^{\prime \prime}\right) \backslash\left\{\phi_{\mu}\left(z, w^{\prime}\right)\right\}\right)$ for any $\left(z, w^{\prime}\right) \in\left(A \cap \Delta_{a}\left(\delta^{\prime}\right)\right) \times \Delta_{b^{\prime}}\left(\delta^{\prime}\right)$. Using the biholomorphic mapping

$$
\begin{aligned}
& \Phi_{\mu}: \Delta_{\left(a, b^{\prime}\right)}\left(\delta^{\prime}\right) \times \mathbb{C} \rightarrow \Delta_{\left(a, b^{\prime}\right)}\left(\delta^{\prime}\right) \times \mathbb{C}, \\
& \Phi_{\mu}\left(z, w^{\prime}, w_{q}\right):=\left(z, w^{\prime}, w_{q}-\phi_{\mu}\left(z, w^{\prime}\right)\right),
\end{aligned}
$$

we see that the function $g:=\widehat{f}_{1} \circ \Phi_{\mu}^{-1}$ is holomorphic in $\Delta_{\left(a, b^{\prime}\right)}\left(\delta^{\prime \prime}\right) \times$ $\left(\Delta_{0}\left(\eta^{\prime \prime}\right) \backslash \bar{\Delta}_{0}\left(\eta^{\prime}\right)\right)$ for some $\delta^{\prime \prime} \in\left(0, \delta^{\prime}\right]$ and $\varepsilon^{\prime}<\eta^{\prime}<\eta^{\prime \prime}<\varepsilon^{\prime \prime}$. Moreover, $g\left(z, w^{\prime}, \cdot\right) \in \mathcal{O}\left(\Delta_{0}\left(\eta^{\prime \prime}\right) \backslash\{0\}\right)$ for any $\left(z, w^{\prime}\right) \in\left(A \cap \Delta_{a}\left(\delta^{\prime \prime}\right)\right) \times \Delta_{b^{\prime}}\left(\delta^{\prime \prime}\right)$. Using Theorem 1 for the cross

$$
\mathbb{X}\left(\left(A \cap \Delta_{a}\left(\delta^{\prime \prime}\right)\right) \times \Delta_{b^{\prime}}\left(\delta^{\prime \prime}\right), \Delta_{0}\left(\eta^{\prime \prime}\right) \backslash \bar{\Delta}_{0}\left(\eta^{\prime}\right) ; \Delta_{\left(a, b^{\prime}\right)}\left(\delta^{\prime \prime}\right), \Delta_{0}\left(\eta^{\prime \prime}\right) \backslash\{0\}\right)
$$


shows that $g$ extends holomorphically to $\Delta_{\left(a, b^{\prime}\right)}\left(\delta^{\prime \prime}\right) \times\left(\Delta_{0}\left(\eta^{\prime \prime}\right) \backslash\{0\}\right)$ (because $\left.h_{\Delta_{0}\left(\eta^{\prime \prime}\right) \backslash\{0\}, \Delta_{0}\left(\eta^{\prime \prime}\right) \backslash \bar{\Delta}_{0}\left(\eta^{\prime}\right)}^{*} \equiv 0\right)$.

Translating the above information back via $\Phi_{\mu}$ for all $\mu$, we conclude that the function $\widehat{f}_{1}$ extends holomorphically to $\Delta_{\left(a, b^{\prime}\right)}\left(\delta^{\prime \prime \prime}\right) \times \Delta_{b_{0, q}}\left(R^{\prime \prime \prime}\right) \backslash M$ for some $\delta^{\prime \prime \prime} \in\left(0, \delta^{\prime \prime}\right]$; in particular, $\widehat{f}_{1}$ extends holomorphically to $\Delta_{\left(a, b^{\prime}\right)}\left(\delta^{\prime \prime \prime}\right)$ $\times \Delta_{b_{0, q}}\left(R_{0}\right) \backslash M$.

Now we prove that $\left(\widetilde{A} \times \Delta_{b_{0}^{\prime}}\left(R^{\prime}\right)\right) \backslash A^{\prime}$ is pluripolar. Write

$$
M \cap\left(\Delta_{a_{0}}\left(\varrho^{\prime}\right) \times \Delta_{b_{0}^{\prime}}\left(R^{\prime}\right) \times \Delta_{b_{0, q}}(R)\right)=\bigcup_{\nu=1}^{\infty}\left\{\zeta \in P_{\nu}: g_{\nu}(\zeta)=0\right\},
$$

where $P_{\nu} \Subset \Delta_{a_{0}}\left(\varrho^{\prime}\right) \times \Delta_{b_{0}^{\prime}}\left(R^{\prime}\right) \times \Delta_{b_{0, q}}(R)$ is a polydisc and $g_{j} \in \mathcal{O}\left(P_{j}\right)$ is a defining function for $M \cap P_{j}$; cf. [Chi 1989, §2.9]. Define

$$
S_{\nu}:=\left\{\zeta=\left(\widetilde{\zeta}, \zeta_{p+q}\right) \in P_{\nu}: g_{\nu}(\zeta)=\frac{\partial g_{\nu}}{\partial \zeta_{p+q}}(\zeta)=0\right\}
$$

and observe that, by the implicit function theorem, any point from

$$
\left(\widetilde{A} \times \Delta_{b_{0}^{\prime}}\left(R^{\prime}\right)\right) \backslash \bigcup_{\nu=1}^{\infty} \operatorname{pr}_{\widetilde{\zeta}}\left(S_{\nu}\right)
$$

satisfies $\left(\begin{array}{l}* \\ *\end{array}\right)$. It is enough to show that each set $\operatorname{pr}_{\tilde{\zeta}}\left(S_{\nu}\right)$ is pluripolar. Fix $\nu$. Let $S$ be an irreducible component of $S_{\nu}$. We have to show that $\operatorname{pr}_{\widetilde{\zeta}}(S)$ is pluripolar. If $S$ has codimension $\geq 2$, then $\operatorname{pr}_{\widetilde{\zeta}}(S)$ is contained in a countable union of proper analytic sets (cf. [Chi 1989, §3.8]). Consequently, $\operatorname{pr}_{\widetilde{\zeta}}(S)$ is pluripolar. Thus we may assume that $S$ is pure one-codimensional. The same argument as above shows that $\operatorname{pr}_{\widetilde{\zeta}}(\operatorname{Sing}(S))$ is pluripolar. It remains to prove that $\operatorname{pr}_{\widetilde{\zeta}}(\operatorname{Reg}(S))$ is pluripolar. Since $g_{\nu}$ is a defining function, for any $\zeta \in \operatorname{Reg}(S)$ there exists a $k \in\{1, \ldots, p+q-1\}$ such that

$$
\frac{\partial g_{\nu}}{\partial \zeta_{k}}(\zeta) \neq 0
$$

Thus

$$
\operatorname{Reg}(S)=\bigcup_{k=1}^{p+q-1} T_{k}
$$

where

$$
T_{k}:=\left\{\zeta \in \operatorname{Reg}(S): \frac{\partial g_{\nu}}{\partial \zeta_{k}}(\zeta) \neq 0\right\} .
$$

We only need to prove that each set $\operatorname{pr}_{\widetilde{\zeta}}\left(T_{k}\right)$ is pluripolar, $k=1, \ldots, p+q-1$. Fix $k$. To simplify notation, assume that $k=1$. Observe that, by the implicit 
function theorem, we can write

$$
T_{1}=\bigcup_{l=1}^{\infty}\left\{\zeta \in Q_{l}: \zeta_{1}=\psi_{l}\left(\zeta_{2}, \ldots, \zeta_{p+q}\right)\right\}
$$

where $Q_{l} \subset P_{\nu}$ is a polydisc, $Q_{l}=Q_{l}^{\prime} \times Q_{l}^{\prime \prime} \subset \mathbb{C} \times \mathbb{C}^{p+q-1}$, and $\psi_{l}: Q_{l}^{\prime \prime} \rightarrow Q_{l}^{\prime}$ is holomorphic, $l \in \mathbb{N}$. It suffices to prove that the projection of each set $T_{1, l}:=\left\{\zeta \in Q_{l}: \zeta_{1}=\psi_{l}\left(\zeta_{2}, \ldots, \zeta_{p+q}\right)\right\}$ is pluripolar. Fix $l$. Since

$$
g_{\nu}\left(\psi_{l}\left(\zeta_{2}, \ldots, \zeta_{p+q}\right), \zeta_{2}, \ldots, \zeta_{p+q}\right)=0, \quad\left(\zeta_{2}, \ldots, \zeta_{p+q}\right) \in Q_{l}^{\prime \prime},
$$

we conclude that $\partial \psi_{l} / \partial \zeta_{p+q} \equiv 0$ and consequently $\psi_{l}$ is independent of $\zeta_{p+q}$. Thus $\operatorname{pr}_{\tilde{\zeta}}\left(T_{1, l}\right)=\left\{\zeta_{1}=\psi_{l}\left(\zeta_{2}, \ldots, \zeta_{p+q-1}\right)\right\}$ and therefore the projection is pluripolar. The proof that $\left(\widetilde{A} \times \Delta_{b_{0}^{\prime}}\left(R^{\prime}\right)\right) \backslash A^{\prime}$ is pluripolar is complete.

Using Step 4, we conclude that $\widehat{f}$ extends holomorphically to the domain $\widehat{Y} \backslash M$, where

$$
\begin{aligned}
& \widehat{Y}:=\left\{\left(z, w^{\prime}, w_{q}\right) \in \Delta_{a_{0}}\left(\varrho^{\prime}\right) \times \Delta_{b_{0}^{\prime}}\left(R^{\prime}\right) \times \Delta_{b_{0, q}}\left(R_{0}\right):\right. \\
&\left.h_{A^{\prime}, \Delta_{a_{0}}\left(\varrho^{\prime}\right) \times \Delta_{b_{0}^{\prime}}\left(R^{\prime}\right)}^{*}\left(z, w^{\prime}\right)+h_{\Delta_{b_{0, q}}\left(R^{\prime}\right), \Delta_{b_{0, q}}\left(R_{0}\right)}^{*}\left(w_{q}\right)<1\right\} \\
&=\left\{\left(z, w^{\prime}, w_{q}\right) \in \Delta_{a_{0}}\left(\varrho^{\prime}\right) \times \Delta_{b_{0}^{\prime}}\left(R^{\prime}\right) \times \Delta_{b_{0, q}}\left(R_{0}\right):\right. \\
&\left.h_{\widetilde{A}, \Delta_{a_{0}}\left(\varrho^{\prime}\right)}^{*}(z)+h_{\Delta_{b_{0, q}}\left(R^{\prime}\right), \Delta_{b_{0, q}}\left(R_{0}\right)}^{*}\left(w_{q}\right)<1\right\}
\end{aligned}
$$

(here we have used the product property of the relative extremal function). Since $R_{0}^{\prime}<R_{0}$, we find a $\varrho_{q} \in\left(0, \varrho^{\prime}\right]$ and a function $\widetilde{f}_{q} \in \mathcal{O}\left(\Delta_{a_{0}}\left(\varrho_{q}\right) \times\right.$ $\left.\Delta_{b_{0}^{\prime}}\left(R^{\prime}\right) \times \Delta_{b_{0, q}}\left(R_{0}^{\prime}\right) \backslash M\right)$ such that

$$
\widetilde{f}_{q}=\widehat{f} \quad \text { on } \Delta_{a_{0}}\left(\varrho_{q}\right) \times \Delta_{b_{0}}\left(R^{\prime}\right) \backslash M .
$$

If $q=1$ we get a contradiction (because $R_{0}^{\prime}>R_{0}^{*}$ ).

Let $q \geq 2$. Repeating the above argument for the coordinates $w_{\nu}, \nu=$ $1, \ldots, q-1$, we find a $\varrho_{0} \in\left(0, \varrho^{\prime}\right]$ and a function $\widetilde{f}$ holomorphic in

$$
\Delta_{a_{0}}\left(\varrho_{0}\right) \times\left(\bigcup_{\nu=1}^{q} \Delta_{\left(b_{0,1}, \ldots, b_{0, \nu-1}\right)}\left(R^{\prime}\right) \times \Delta_{b_{0, \nu}}\left(R_{0}^{\prime}\right) \times \Delta_{\left(b_{0, \nu+1}, \ldots, b_{0, q}\right)}\left(R^{\prime}\right)\right) \backslash M
$$

such that $\tilde{f}=\widehat{f}$ on $\Delta_{a_{0}}\left(\varrho_{0}\right) \times \Delta_{b_{0}}\left(R^{\prime}\right) \backslash M$. Let $\mathcal{H}$ denote the envelope of holomorphy of the domain

$$
\bigcup_{\nu=1}^{q} \Delta_{\left(b_{0,1}, \ldots, b_{0, \nu-1}\right)}\left(R^{\prime}\right) \times \Delta_{b_{0, \nu}}\left(R_{0}^{\prime}\right) \times \Delta_{\left(b_{0, \nu+1}, \ldots, b_{0, q}\right)}\left(R^{\prime}\right) .
$$

Applying the Grauert-Remmert theorem, we can extend $\widetilde{f}$ holomorphically to $\Delta_{a_{0}}\left(\varrho_{0}\right) \times \mathcal{H} \backslash M$, i.e. there exists an $\widehat{\widetilde{f}} \in \mathcal{O}\left(\Delta_{a_{0}}\left(\varrho_{0}\right) \times \mathcal{H} \backslash M\right)$ with $\widehat{\widetilde{f}}=f$ on $\Delta_{a_{0}}\left(\varrho_{0}\right) \times \Delta_{b_{0}}(r)$. Observe that $\Delta_{b_{0}}\left(\sqrt[q]{{R^{\prime}}^{q-1} R_{0}^{\prime}}\right) \subset \mathcal{H}$. Recall that $\sqrt[q]{R^{\prime q-1} R_{0}^{\prime}}>R_{0}^{*} ;$ a contradiction. 
REMARK. Notice that the proof of Step 6 shows that the following stronger version of $(*)$ is true: Let $\varrho>0,0<r<R, \Omega, \widetilde{\Omega}, A$, and $a$ be as in (*). Let $M$ be a pure one-codimensional analytic subset of $\Omega$ (we do not assume that $M \cap \widetilde{\Omega}=\emptyset)$. Then:

For any $R^{\prime} \in(r, R)$ there exists $\varrho^{\prime} \in(0, \varrho)$ such that for any function $f \in$ $\mathcal{O}(\widetilde{\Omega} \backslash M)$ with $f(a, \cdot) \in \mathcal{O}\left(\Delta_{b_{0}}(R) \backslash M_{a}\right), a \in A$, there exists an extension $\widehat{f} \in \mathcal{O}\left(\Delta_{a_{0}}\left(\varrho^{\prime}\right) \times \Delta_{b_{0}}\left(R^{\prime}\right) \backslash M\right)$ with $\widehat{f}=f$ on $\Delta_{a_{0}}\left(\varrho^{\prime}\right) \times \Delta_{b_{0}}(r) \backslash M$.

4. Proof of the Main Theorem in the general case. First observe that the function $\widehat{f}$ is uniquely determined (cf. $\S 3$ ).

We proceed by induction on $N$. Let $D_{j, k} \nearrow D_{j}, D_{j, k} \Subset D_{j, k+1} \Subset D_{j}$, where $D_{j, k}$ are pseudoconvex domains with $A_{j, k}:=A_{j} \cap D_{j, k} \neq \emptyset$. Put

$$
X_{k}:=\mathbb{X}\left(A_{1, k}, \ldots, A_{N, k} ; D_{1, k}, \ldots, D_{N, k}\right) \subset X .
$$

It suffices to show that for each $k \in \mathbb{N}$ the following condition $(\stackrel{* *}{*})$ holds:

$(\stackrel{* *}{*}) \quad$ There exists a domain $U_{k}, X_{k} \subset U_{k} \subset U \cap \widehat{X}_{k}$, such that for any $f \in$ $\mathcal{O}_{\mathrm{s}}(X \backslash M)$ there exists an $\widetilde{f}_{k} \in \mathcal{O}\left(U_{k} \backslash M\right)$ with $\left.\widetilde{f}_{k}\right|_{X_{k} \backslash M}=\left.f\right|_{X_{k} \backslash M}$.

Indeed, fix $k \in \mathbb{N}$ and observe that $\widehat{X}_{k}$ is the envelope of holomorphy of $U_{k}$ (cf. the proof of Step 4). Hence, by the Dloussky theorem (cf. [Jar-Pfl 2000, Th. 3.4.8], see also [Por 2002]), there exists an analytic subset $\widetilde{M}_{k}$ of $\widehat{X}_{k}, \widetilde{M}_{k} \cap U_{k} \subset M$, such that $\widehat{X}_{k} \backslash \widetilde{M}_{k}$ is the envelope of holomorphy of $U_{k} \backslash M$. In particular, for each $f \in \mathcal{O}_{\mathrm{s}}(X \backslash M)$ there exists an $\widetilde{\widetilde{f}}_{k} \in \mathcal{O}\left(\widehat{X}_{k} \backslash \widetilde{M}_{k}\right)$ with $\left.\widetilde{\widetilde{f}}_{k}\right|_{U_{k} \backslash M}=\widetilde{f}_{k}$. Let $\mathcal{F}_{k}:=\left\{\widetilde{\widetilde{f}}_{k}: f \in \mathcal{O}_{\mathrm{s}}(X \backslash M)\right\} \subset \mathcal{O}\left(\widehat{X}_{k} \backslash \widetilde{M}_{k}\right)$. It is known (cf. [Jar-Pfl 2000, Prop. 3.4.5]) that there exists a pure one-codimensional analytic subset $\widehat{M}_{k} \subset \widehat{X}_{k}, \widehat{M}_{k} \subset \widetilde{M}_{k}$, such that any point of $\widehat{M}_{k}$ is singular with respect to $\mathcal{F}_{k}$, i.e.

- any function $\widetilde{\widetilde{f}}_{k}$ extends to a function $\widehat{f}_{k} \in \mathcal{O}\left(\widehat{X}_{k} \backslash \widehat{M}_{k}\right)$, and

- for any $a \in \widehat{M}_{k}$ and an open neighborhood $V$ of $a, V \subset \widehat{X}_{k}$, there exists an $f \in \mathcal{O}_{\mathrm{s}}(X \backslash M)$ such that $\left.\widehat{f}_{k}\right|_{V \backslash \widehat{M}_{k}}$ cannot be holomorphically extended to the whole $V$.

In particular, $\widehat{M}_{k+1} \cap \widehat{X}_{k}=\widehat{M}_{k}$. Consequently, $\widehat{M}:=\bigcup_{k=1}^{\infty} \widehat{M}_{k}$ is a pure one-codimensional analytic subset of $\widehat{X}, \widehat{M} \cap \bigcup_{k=1}^{\infty} U_{k} \subset M$, and for each $f \in \mathcal{O}_{\mathrm{s}}(X \backslash M)$, the function $\widehat{f}:=\bigcup_{k=1}^{\infty} \widehat{f_{k}}$ is holomorphic on $\widehat{X} \backslash \widehat{M}$ with $\left.\widehat{f}\right|_{X \backslash M}=f$.

It remains to prove $\left(\begin{array}{c}* * \\ *\end{array}\right)$. Fix $k \in \mathbb{N}$. For any $a=\left(a_{1}, \ldots, a_{N}\right) \in A_{1, k} \times$ $\ldots \times A_{N, k}$ let $\varrho=\varrho_{k}(a)$ be such that $\Delta_{a}(\varrho) \subset D_{1, k} \times \ldots \times D_{N, k}$. If $N \geq 4$, 
then we additionally define $(N-2)$-fold crosses $Y_{k, \mu, \nu}:=\mathbb{X}\left(A_{1, k}, \ldots, A_{\mu-1, k}, A_{\mu+1, k}, \ldots, A_{\nu-1, k}, A_{\nu+1, k}, \ldots, A_{N, k} ;\right.$ $\left.D_{1, k}, \ldots, D_{\mu-1, k}, D_{\mu+1, k}, \ldots, D_{\nu-1, k}, D_{\nu+1, k}, \ldots, D_{N, k}\right), \quad 1 \leq \mu<\nu \leq N$, and we assume that $\varrho$ is so small that

$$
\Delta_{\left(a_{1}, \ldots, a_{\mu-1}, a_{\mu+1}, \ldots, a_{\nu-1}, a_{\nu+1}, \ldots, a_{N}\right)}(\varrho) \subset \widehat{Y}_{k, \mu, \nu}, \quad 1 \leq \mu<\nu \leq N .
$$

Since $\left\{\left(a_{1}, \ldots, a_{j-1}\right)\right\} \times \bar{D}_{j, k+1} \times\left\{\left(a_{j+1}, \ldots, a_{N}\right)\right\} \Subset U$, we may assume that

$$
\Delta_{\left(a_{1}, \ldots, a_{j-1}\right)}(\varrho) \times D_{j, k+1} \times \Delta_{\left(a_{j+1}, \ldots, a_{N}\right)}(\varrho) \subset U, \quad j=1, \ldots, N .
$$

We define $N$-fold crosses

$$
\begin{aligned}
Z_{k, a, j}:=\mathbb{X} & \left(A_{1} \cap \Delta_{a_{1}}(\varrho), \ldots, A_{j-1} \cap \Delta_{a_{j-1}}(\varrho), A_{j, k+1},\right. \\
& A_{j+1} \cap \Delta_{a_{j+1}}(\varrho), \ldots, A_{N} \cap \Delta_{a_{N}}(\varrho) \\
& \left.\Delta_{a_{1}}(\varrho), \ldots, \Delta_{a_{j-1}}(\varrho), D_{j, k+1}, \Delta_{a_{j+1}}(\varrho), \ldots, \Delta_{a_{N}}(\varrho)\right)
\end{aligned}
$$

for $j=1, \ldots, N$. Note that $\widehat{Z}_{k, a, j} \subset U$. Since $\left\{\left(a_{1}, \ldots, a_{j-1}\right)\right\} \times \bar{D}_{j, k} \times$ $\left\{\left(a_{j+1}, \ldots, a_{N}\right)\right\} \Subset \widehat{Z}_{k, a, j}$, there exists an $r=r_{k}(a), 0<r \leq \varrho$, so small that

$$
V_{k, a, j}:=\Delta_{\left(a_{1}, \ldots, a_{j-1}\right)}(r) \times D_{j, k} \times \Delta_{\left(a_{j+1}, \ldots, a_{N}\right)}(r) \subset \widehat{Z}_{k, a, j}, \quad j=1, \ldots, N .
$$

Put

$$
V_{k}:=\bigcup_{\substack{a \in A_{1, k} \times \ldots \times A_{N, k} \\ j \in\{1, \ldots, N\}}} V_{k, a, j} .
$$

Note that $X_{k} \subset V_{k}$. Let $U_{k}$ be the connected component of $V_{k} \cap \widehat{X}_{k}$ that contains $X_{k}$.

In view of (4), the Main Theorem with $U=\widehat{X}$ (which is already proved in $\S 3)$ implies that for any $f \in \mathcal{O}_{\mathrm{s}}(X \backslash M)$ there exists an extension $\widehat{f}_{k, a, j} \in$ $\mathcal{O}\left(\widehat{Z}_{k, a, j} \backslash M\right)$ of $\left.f\right|_{Z_{k, a, j} \backslash M}$. It remains to glue the functions

$$
\widetilde{f}_{k, a, j}:=\left.\widehat{f}_{k, a, j}\right|_{V_{k, a, j} \backslash M}, \quad a \in A_{1, k} \times \ldots \times A_{N, k}, \quad j=1, \ldots, N ;
$$

then the function

$$
\widetilde{f}_{k}:=\left.\left(\bigcup_{\substack{a \in A_{1, k} \times \ldots \times A_{N, k} \\ j \in\{1, \ldots, N\}}} \tilde{f}_{k, a, j}\right)\right|_{U_{k} \backslash M}
$$

gives the required extension of $\left.f\right|_{X_{k} \backslash M}$.

To check that the gluing process is possible, let $a, b \in A_{1, k} \times \ldots \times A_{N, k}$ and $i, j \in\{1, \ldots, N\}$ be such that $V_{k, a, i} \cap V_{k, b, j} \neq \emptyset$. We have the following two cases: 
(a) $i \neq j$ : We may assume that $i=N-1, j=N$. Write $w=\left(w^{\prime}, w^{\prime \prime}\right) \in$ $\mathbb{C}^{k_{1}+\ldots+k_{N-2}} \times \mathbb{C}^{k_{N-1}+k_{N}}$. Observe that

$V_{k, a, N-1} \cap V_{k, b, N}=\left(\Delta_{a^{\prime}}\left(r_{k}(a)\right) \cap \Delta_{b^{\prime}}\left(r_{k}(b)\right)\right) \times \Delta_{b_{N-1}}\left(r_{k}(b)\right) \times \Delta_{a_{N}}\left(r_{k}(a)\right)$.

For $c=\left(c^{\prime}, c^{\prime \prime}\right)$, let

$$
\begin{aligned}
M_{c^{\prime}} & :=\left\{w^{\prime \prime} \in \mathbb{C}^{k_{N-1}+k_{N}}:\left(c^{\prime}, w^{\prime \prime}\right) \in M\right\}, \\
M^{c^{\prime \prime}} & :=\left\{w^{\prime} \in \mathbb{C}^{k_{1}+\ldots+k_{N-2}}:\left(w^{\prime}, c^{\prime \prime}\right) \in M\right\} ;
\end{aligned}
$$

$M_{c^{\prime}}$ and $M^{c^{\prime \prime}}$ are analytic subsets of

$$
\begin{aligned}
U_{c^{\prime}} & :=\left\{w^{\prime \prime} \in \mathbb{C}^{k_{N-1}+k_{N}}:\left(c^{\prime}, w^{\prime \prime}\right) \in U\right\}, \\
U^{c^{\prime \prime}} & :=\left\{w^{\prime} \in \mathbb{C}^{k_{1}+\ldots+k_{N-2}}:\left(w^{\prime}, c^{\prime \prime}\right) \in U\right\},
\end{aligned}
$$

respectively.

We consider the following three subcases:

$\left(\mathrm{a}_{1}\right) N=2$ : Then $V_{k, a, 1} \cap V_{k, b, 2}=\Delta_{b_{1}}\left(r_{k}(b)\right) \times \Delta_{a_{2}}\left(r_{k}(a)\right)$. Since $\widetilde{f}_{k, a, 1}=$ $\widetilde{f}_{k, b, 2}$ on the non-pluripolar set $\left(A_{1} \cap \Delta_{b_{1}}\left(r_{k}(b)\right)\right) \times\left(A_{2} \cap \Delta_{a_{2}}\left(r_{k}(a)\right)\right) \backslash M$, by the identity principle, $\widetilde{f}_{k, a, 1}=\widetilde{f}_{k, b, 2}$ on $V_{k, a, 1} \cap V_{k, b, 2} \backslash M$.

$\left(\mathrm{a}_{2}\right) N=3$ : Then $V_{k, a, 2} \cap V_{k, b, 3}=\left(\Delta_{a_{1}}\left(r_{k}(a)\right) \cap \Delta_{b_{1}}\left(r_{k}(b)\right)\right) \times \Delta_{b_{2}}\left(r_{k}(b)\right)$ $\times \Delta_{a_{3}}\left(r_{k}(a)\right)$. Let $C^{\prime \prime}$ denote the set of all points $c^{\prime \prime} \in\left(A_{2} \cap \Delta_{b_{2}}\left(r_{k}(b)\right)\right) \times$ $\left(A_{3} \cap \Delta_{a_{3}}\left(r_{k}(a)\right)\right)$ such that the set $M^{c^{\prime \prime}}$ has codimension $\geq 1$ (i.e. for any $w^{\prime} \in M^{c^{\prime \prime}}$ the codimension of $M^{c^{\prime \prime}}$ at $w^{\prime}$ is $\left.\geq 1\right)$. Note that $C^{\prime \prime}$ is nonpluripolar. We have $\widetilde{f}_{k, a, 2}\left(\cdot, c^{\prime \prime}\right)=f\left(\cdot, c^{\prime \prime}\right)=\widetilde{f}_{k, b, 3}\left(\cdot, c^{\prime \prime}\right)$ on $\Delta_{a_{1}}\left(r_{k}(a)\right) \cap$ $\Delta_{b_{1}}\left(r_{k}(b)\right) \backslash M^{c^{\prime \prime}}$.

Now, let $c^{\prime} \in \Delta_{a_{1}}\left(r_{k}(a)\right) \cap \Delta_{b_{1}}\left(r_{k}(b)\right)$ be such that the set $M_{c^{\prime}}$ has codimension $\geq 1$. Then $\widetilde{f}_{k, a, 2}\left(c^{\prime}, \cdot\right)=\widetilde{f}_{k, b, 3}\left(c^{\prime}, \cdot\right)$ on $C^{\prime \prime} \backslash M_{c^{\prime}}$. Hence, by the identity principle, $\widetilde{f}_{k, a, 2}\left(c^{\prime}, \cdot\right)=\widetilde{f}_{k, b, 3}\left(c^{\prime}, \cdot\right)$ on $\Delta_{b_{2}}\left(r_{k}(b)\right) \times \Delta_{a_{3}}\left(r_{k}(a)\right) \backslash M_{c^{\prime}}$. Finally, $\widetilde{f}_{k, a, 2}=\widetilde{f}_{k, b, 3}$ on $V_{k, a, 2} \cap V_{k, b, 3} \backslash M$.

If $N \in\{2,3\}$, then we jump directly to (b) and we conclude that the Main Theorem is true for $N \in\{2,3\}$.

(a) $N \geq 4$ : Here is the only place where the induction over $N$ is used. We assume that the Main Theorem is true for $N-1 \geq 3$.

Similarly to the case $N=3$, let $C^{\prime \prime}$ denote the set of all points $c^{\prime \prime} \in$ $\left(A_{N-1} \cap \Delta_{b_{N-1}}\left(r_{k}(b)\right)\right) \times\left(A_{N} \cap \Delta_{a_{N}}\left(r_{k}(a)\right)\right)$ such that the set $M^{c^{\prime \prime}}$ has codimension $\geq 1$; $C^{\prime \prime}$ is non-pluripolar. The function $f_{c^{\prime \prime}}:=f\left(\cdot, c^{\prime \prime}\right)$ is separately holomorphic on $Y_{k, N-1, N} \backslash M^{c^{\prime \prime}}$. By the inductive assumption, $f_{c^{\prime \prime}}$ extends to a function $\widehat{f}_{c^{\prime \prime}} \in \mathcal{O}\left(\widehat{Y}_{k, N-1, N} \backslash \widehat{M}\left(c^{\prime \prime}\right)\right)$, where $\widehat{M}\left(c^{\prime \prime}\right)$ is an analytic subset of $\widehat{Y}_{k, N-1, N}$ with $\widehat{M}\left(c^{\prime \prime}\right) \subset M^{c^{\prime \prime}}$ in an open neighborhood of 
$Y_{k, N-1, N} \cdot$ Recall that

$$
\Delta_{a^{\prime}}\left(r_{k}(a)\right) \cup \Delta_{b^{\prime}}\left(r_{k}(b)\right) \subset \widehat{Y}_{k, N-1, N} .
$$

Since $\widetilde{f}_{k, a, N-1}\left(\cdot, c^{\prime \prime}\right)=f_{c^{\prime \prime}}$ on $\Delta_{a^{\prime}}\left(r_{k}(a)\right) \cap Y_{k, N-1, N} \backslash M^{c^{\prime \prime}}$ and $\widetilde{f}_{k, b, N}\left(\cdot, c^{\prime \prime}\right)=$ $f_{c^{\prime \prime}}$ on $\Delta_{b^{\prime}}\left(r_{k}(b)\right) \cap Y_{k, N-1, N} \backslash M^{c^{\prime \prime}}$, we conclude that $\widetilde{f}_{k, a, N-1}\left(\cdot, c^{\prime \prime}\right)=\widehat{f}_{c^{\prime \prime}}=$ $\widetilde{f}_{k, b, N}\left(\cdot, c^{\prime \prime}\right)$ on $\Delta_{a^{\prime}}\left(r_{k}(a)\right) \cap \Delta_{b^{\prime}}\left(r_{k}(b)\right) \backslash M^{c^{\prime \prime}}$.

Let $c^{\prime} \in \Delta_{a^{\prime}}\left(r_{k}(a)\right) \cap \Delta_{b^{\prime}}\left(r_{k}(b)\right)$ be such that the set $M_{c^{\prime}}$ has codimension $\geq 1$. Then $\widetilde{f}_{k, a, N-1}\left(c^{\prime}, \cdot\right)=\widetilde{f}_{k, b, N}\left(c^{\prime}, \cdot\right)$ on $C^{\prime \prime} \backslash M_{c^{\prime}}$. Consequently, by the identity principle, $\widetilde{f}_{k, a, N-1}\left(c^{\prime}, \cdot\right)=\widetilde{f}_{k, b, N}\left(c^{\prime}, \cdot\right)$ on $\Delta_{b_{N-1}}\left(r_{k}(b)\right) \times$ $\Delta_{a_{N}}\left(r_{k}(a)\right) \backslash M_{c^{\prime}}$ and, finally, $\widetilde{f}_{k, a, N-1}=\widetilde{f}_{k, b, N}$ on $V_{k, a, N-1} \cap V_{k, b, N} \backslash M$.

(b) $i=j$ : We may assume that $i=j=N$. Observe that

$$
V_{k, a, N} \cap V_{k, b, N}=\left(\Delta_{\left(a_{1}, \ldots, a_{N-1}\right)}\left(r_{k}(a)\right) \cap \Delta_{\left(b_{1}, \ldots, b_{N-1}\right)}\left(r_{k}(b)\right)\right) \times D_{N, k} .
$$

By (a) we know that

$$
\begin{aligned}
\widetilde{f}_{k, a, N} & =\widetilde{f}_{k, a, N-1} & & \text { on } V_{k, a, N} \cap V_{k, a, N-1} \backslash M, \\
\widetilde{f}_{k, a, N-1} & =\widetilde{f}_{k, b, N} & & \text { on } V_{k, a, N-1} \cap V_{k, b, N} \backslash M .
\end{aligned}
$$

Hence $\widetilde{f}_{k, a, N}=\widetilde{f}_{k, b, N}$ on

$$
\begin{aligned}
& V_{k, a, N} \cap V_{k, a, N-1} \cap V_{k, b, N} \backslash M \\
& \quad=\left(\Delta_{\left(a_{1}, \ldots, a_{N-1}\right)}\left(r_{k}(a)\right) \cap \Delta_{\left(b_{1}, \ldots, b_{N-1}\right)}\left(r_{k}(b)\right)\right) \times \Delta_{a_{N}}\left(r_{k}(a)\right) \backslash M,
\end{aligned}
$$

and finally, by the identity principle,

$$
\widetilde{f}_{k, a, N}=\widetilde{f}_{k, b, N} \quad \text { on } V_{k, a, N} \cap V_{k, b, N} \backslash M .
$$

The proof of the Main Theorem is complete.

\section{References}

[Ale-Zer 2001] O. Alehyane et A. Zeriahi, Une nouvelle version du théorème d'extension de Hartogs pour les applications séparément holomorphes entre espaces analytiques, Ann. Polon. Math. 76 (2001), 245-278.

[Chi 1989] E. M. Chirka, Complex Analytic Sets, Kluwer, 1989.

[Jar-Pfl 2000] M. Jarnicki and P. Pflug, Extension of Holomorphic Functions, de Gruyter Exp. Math. 34, de Gruyter, 2000.

[Jar-Pfl 2001] —, —, Cross theorem, Ann. Polon. Math. 77 (2001), 295-302.

[Jar-Pfl 2003] —, - An extension theorem for separately holomorphic functions with pluripolar singularities, Trans. Amer. Math. Soc. 355 (2003), 1251-1267.

[Kli 1991] M. Klimek, Pluripotential Theory, Oxford Univ. Press, 1991.

[Ngu 1997] Nguyen Thanh Van, Separate analyticity and related subjects, Vietnam J. Math. 25 (1997), 81-90.

[Ngu-Sic 1991] Nguyen Thanh Van et J. Siciak, Fonctions plurisousharmoniques extrémales et systèmes doublement orthogonaux de fonctions analytiques, Bull. Sci. Math. 115 (1991), 235-243. 
[Ngu-Zer 1991] Nguyen Thanh Van et A. Zeriahi, Une extension du théorème de Hartogs sur les fonctions séparément analytiques, in: Analyse Complexe Multivariable, Récents Développements, A. Meril (ed.), EditEl, Rende, 1991, 183-194.

[Ngu-Zer 1995] —, 一, Systèmes doublement orthogonaux de fonctions holomorphes et applications, in: Banach Center Publ. 31, Inst. Math., Polish Acad. Sci., 1995, 281-297.

[Ökt 1998] O. Öktem, Extension of separately analytic functions and applications to range characterization of the exponential Radon transform, Ann. Polon. Math. 70 (1998), 195-213.

[Ökt 1999] - Extending separately analytic functions in $\mathbb{C}^{n+m}$ with singularities, in: Extension of separately analytic functions and applications to mathematical tomography, thesis, Dept. Math., Stockholm Univ., 1999.

[Por 2002] E. Porten, On the Hartogs-phenomenon and extension of analytic hypersurfaces in non-separated Riemann domains, Complex Variables Theory Appl. 47 (2002), 325-332.

[Shi 1989] B. Shiffman, On separate analyticity and Hartogs theorems, Indiana Univ. Math. J. 38 (1989), 943-957.

[Sic 1969] J. Siciak, Separately analytic functions and envelopes of holomorphy of some lower dimensional subsets of $\mathbb{C}^{n}$, Ann. Polon. Math. 22 (19691970), 147-171.

[Sic 1981] - Extremal plurisubharmonic functions in $\mathbb{C}^{N}$, ibid. 39 (1981), 175211.

[Sic 2001] - Holomorphic functions with singularities on algebraic sets, Univ. Iagel. Acta Math. 39 (2001), 9-16.

[Zah 1976] V. P. Zahariuta, Separately analytic functions, generalizations of Hartogs theorem, and envelopes of holomorphy, Math. USSR-Sb. 30 (1976), 5167 .

Institute of Mathematics

Jagiellonian University

Reymonta 4

30-059 Kraków, Poland

E-mail: jarnicki@im.uj.edu.pl
Fachbereich Mathematik Carl von Ossietzky Universität Oldenburg Postfach 2503

D-26111 Oldenburg, Germany E-mail: pflug@mathematik.uni-oldenburg.de 